PROCEEDINGS OF THE

AMERICAN MATHEMATICAL SOCIETY

Volume 139, Number 6, June 2011, Pages 1955-1960

S 0002-9939(2010)10642-1

Article electronically published on November 10, 2010

\title{
ON THE AZUMAYA LOCUS OF ALMOST COMMUTATIVE ALGEBRAS
}

\author{
AKAKI TIKARADZE
}

(Communicated by Gail R. Letzter)

\begin{abstract}
We prove a general statement which implies the coincidence of the Azumaya and smooth loci of the center of an algebra in positive characteristic, provided that the spectrum of its associated graded algebra has a large symplectic leaf. In particular, we show that for a symplectic reflection algebra, the smooth and the Azumaya loci coincide.
\end{abstract}

\section{INTRODUCTION}

Throughout, we will fix a ground field $\mathbf{k}$, which will be assumed to be algebraically closed with positive characteristic $p$. In studying the representation theory of an associative k-algebra $A$ with a large center (i.e. $A$ is a finitely generated module over its center), it is important to understand the Azumaya locus of $A$. Recall that the Azumaya locus of $A$ is defined to be the subset of $\operatorname{Spec} \mathbf{Z}(A)$ (where $\mathbf{Z}(A)$ denotes the center of $A$ ) consisting of all prime ideals $I \in \operatorname{Spec} \mathbf{Z}(A)$ such that the localized algebra $A_{I}$ is an Azumaya algebra. If $A$ is a prime Noetherian ring with its center $\mathbf{Z}(A)$ being finitely generated over $\mathbf{k}$, then a character $\chi: \mathbf{Z}(A) \rightarrow k$ belongs to the Azumaya locus if and only if $A_{\chi}=A \otimes_{\mathbf{z}(A)} \mathbf{k}$ affords an irreducible representation whose dimension is the largest possible dimension for an irreducible $A$-module, and this largest possible dimension is the PI-degree of $A$ ( $[\mathrm{BG}$, Proposition 3.1). In particular, the square of the PI-degree of $A$ is equal to the rank of $A$ over $\mathbf{Z}(A)$. It is well known that if the algebra $A$ is smooth, then the Azumaya locus is contained in the smooth locus of $\operatorname{Spec} \mathbf{Z}(A)[\mathrm{BG}$. Thus, the natural question is when are these two open subsets of $\operatorname{Spec} \mathbf{Z}(A)$ equal. In this direction, there is a general result (which will be crucial for us) due to Brown and Goodearl ([BG], Theorem 3.8), which states that if $A$ is a prime Noetherian ring which is Auslander-regular and Cohen-Macaulay, such that the complement of the Azumaya locus in $\operatorname{Spec} \mathbf{Z}(A)$ has codimension at least 2, then the Azumaya and smooth loci coincide. Using this result, Brown and Goodearl [BG] showed that the Azumaya locus coincides with the smooth locus when $A$ is either the universal enveloping algebra of a reductive Lie algebra in a very good characteristic or a quantized algebra of functions or quantized enveloping algebras at roots of unity. Also, Brown and Changtong $[\mathrm{BC}]$ have proved a similar result for rational Cherednik algebras $[\mathrm{BC}]$, and we have shown an analogous result for infinitesimal Hecke algebras of $\mathfrak{s l}_{2}[\mathrm{~T}]$.

Received by the editors November 24, 2009 and, in revised form, April 18, 2010 and June 2, 2010.

2010 Mathematics Subject Classification. Primary 17B35.

(C)2010 American Mathematical Society Reverts to public domain 28 years from publication 
Given the examples above, the natural question is what do the above examples have in common, and whether there is a general condition which will imply that the Azumaya and smooth loci coincide. In this paper we give such a statement. Namely, we will show that if the algebra $A$ can be equipped with a positive filtration such that the associated graded algebra has an open symplectic leaf whose complement has codimension greater than or equal to 2, then the Azumaya locus of $A$ has the complement of codimension at least 2 . The combination of this with the abovementioned result of Brown-Goodearl enables us to state a general result about the coincidence of the Azumaya and smooth loci; in particular, we will show that for a symplectic reflection algebra the Azumaya locus coincides with the smooth locus.

\section{General Results}

Let $A$ be an associative algebra over $\mathbf{k}$ equipped with a nonnegative filtration $A_{n} \subset A_{n+1} \subset \cdots, n \in \mathbb{Z}_{+}$such that the associated graded algebra gr $A$ is commutative. Let $d$ be the largest number such that $\left[A_{n}, A_{m}\right] \subset A_{n+m-d}$ for all $n, m$. Then gr $A$ becomes equipped with the following natural Poisson bracket: for any homogeneous elements $a \in \operatorname{gr} A_{n}, b \in \operatorname{gr} A_{m}$, one defines their Poisson bracket $\{a, b\}$ to be the symbol of $\left[a^{\prime}, b^{\prime}\right] \in \operatorname{gr} A_{n+m-d}$, where $a^{\prime} \in A_{n}, b^{\prime} \in A_{m}$ are arbitrary lifts of $a, b$. Similarly, if $A$ is a flat associative algebra over $\mathbf{k}[t]$, such that $A / t A$ is commutative, then the commutator bracket of $A$ induces a Poisson bracket on $A / t A$ in the standard way: If $d$ is the largest integer such that $A / t A$ is commutative, then for any $a, b \in A / t A$, one puts $\{a, b\}=\frac{1}{t^{d}}\left[a^{\prime}, b^{\prime}\right]$, where $a^{\prime}, b^{\prime}$ are arbitrary lifts of $a, b$ in $A$.

We start by recalling some terminology and a result by Bezrukavnikov and Kaledin [BK], which will play a crucial role in our proof.

Definition 2.1 ( $[\mathrm{BK}]$ ). A central quantization of a (commutative) Poisson algebra $B$ is a flat associative $\mathbf{k}[[t]]$-algebra $B^{\prime}$, such that $B=B^{\prime} / t B^{\prime}$ and the Poisson bracket of $B$ is induced from the commutator bracket of $B$, and the quotient map from the center of $B^{\prime}$ to the Poisson center of $B$ is surjective.

Here is the result that we are going to use.

Theorem 2.1 ( $\mathrm{BK}$, Proposition 1.24). If under the assumptions of the above definition, Spec $B$ is a symplectic variety, then $B^{\prime}\left[t^{-1}\right]$ is a simple algebra over its center.

The following result will be crucial.

Theorem 2.2. Let $A$ be an associative algebra over $\mathbf{k}$ equipped with an increasing algebra filtration $\mathbf{k}=A_{0} \subset A_{1} \subset \cdots$, such that gr $A$ is a commutative finitely generated domain, and the smooth locus of $X=\operatorname{Spec} g r A, U \subset X$ is a symplectic variety with respect to the Poisson bracket of gr $A$ and the codimension of the singular locus $Z=X-U$ in $X$ is $\geq 2$. If $\operatorname{gr} \mathbf{Z}(A)=(\operatorname{gr} A)^{p}$, then the complement of the Azumaya locus of $\mathbf{Z}(A)$ inside the smooth locus of $\mathbf{Z}(A)$ has codimension $\geq 2$.

Proof. Let $f \in(\operatorname{gr} A)^{p}$ be a nonzero homogeneous element which vanishes on the singular locus of Spec gr $A$, so $f(Z)=0$. Thus, by the assumption, $\operatorname{Spec}\left(\operatorname{gr} A_{f}\right)$ is a symplectic variety. Let us consider an element $g \in \mathbf{Z}(A)$ such that $\sigma(g)=f$ (from now on $\sigma$ will denote the principal part of an element with respect to the filtration). Let us put $S=A_{g}, \operatorname{deg}(g)=d$, where $d$ is a positive integer. Let us consider an 
induced filtration on $A_{g}$ coming from the filtration on $A$, namely $\operatorname{deg} g^{-1}=-d$. Then, gr $S=(\operatorname{gr} A)_{f}$. Let us consider the Rees algebra of $S: R(S)=\sum S_{m} t^{m} \subset$ $S\left[t, t^{-1}\right]$, where $S_{m}$ denotes the set of elements of $S$ of filtration degree $\leq m$. Clearly $R(A)$ is a finitely generated module over its center $\mathbf{Z}(R(A))=R(\mathbf{Z}(A)$ ) (since $R(A)$ is positively graded), and since $R(S)$ is the localization $R(A)$ by a central element $g t^{d}$, we see that $R(S)$ is finite over its center $\mathbf{Z}(R(S))$.

Let us complete $R(S)$ with respect to $t \in R(S)$. Denote this completion by $\overline{R(S)}$, so $\overline{R(S)}=\lim R(S) / t^{n} R(S)$. We have that $\overline{R(S)}$ is a flat module over $k[[t]], \overline{R(S)} / t \overline{R(S)}=(\operatorname{gr} A)_{f}$. Thus by Theorem $\left[2.1, \overline{R(S)}\left[t^{-1}\right]\right.$ is simple over $\overline{R(\mathbf{Z}(S))}\left[t^{-1}\right]$. Notice that $\overline{R(S)}=R(S) \otimes_{\mathbf{Z}(R(S))} \overline{R(\mathbf{Z}(S))}$, so

$$
\overline{R(S)}\left[t^{-1}\right]=R(S)\left[t^{-1}\right] \otimes \mathbf{Z}(R(S))\left[t^{-1}\right] \overline{R(\mathbf{Z}(S))}\left[t^{-1}\right] .
$$

But, $R(S)\left[t^{-1}\right]=S\left[t, t^{-1}\right]$, so we see that $\overline{R(S)}\left[t^{-1}\right]=S \otimes_{\mathbf{Z}(S)} \overline{R(\mathbf{Z}(S))}\left[t^{-1}\right]$, where we use the embedding $i: \mathbf{Z}(S) \rightarrow \overline{R(\mathbf{Z}(S))}\left[t^{-1}\right]$. Thus, we see that if $I \in \operatorname{Spec} \mathbf{Z}(A)$ is a prime ideal of height 1 in the smooth locus of $\mathbf{Z}(A)$ such that $g \notin I$ and $I$ belongs to the image of $i^{*}: \operatorname{Spec} \overline{R(\mathbf{Z}(S))}\left[t^{-1}\right] \rightarrow \operatorname{Spec} \mathbf{Z}(A)$, then there is a faithfully flat base change $\mathbf{Z}(A)_{I} \rightarrow B$, where $B=\overline{R(\mathbf{Z}(S))}\left[t^{-1}\right]_{J}, J \cap \mathbf{Z}(A)=I$, such that $A_{I} \otimes_{\mathbf{Z}(A)_{I}} B$ is a simple algebra over $B$. Therefore, $A_{I}$ is simple over $\mathbf{Z}(A)_{I}$. Since $\mathbf{Z}(A)_{I}$ is a regular local ring of dimension 1 and $A_{I}$ is torsion-free over $\mathbf{Z}(A)_{I}$, we get that $A_{I}$ is projective over $\mathbf{Z}(A)_{I}$. Therefore, $A_{I}$ is Azumaya, so $I$ belongs to the Azumaya locus of $\mathbf{Z}(A)$.

Since we want to show that all primes of height 1 from the smooth locus of $\mathbf{Z}(A)$ belong to the Azumaya locus, it is enough to show that for any such prime $I \in$ $\operatorname{Spec} \mathbf{Z}(A)$, there exists $f \in(\operatorname{gr} A)^{p}$ such that $f(Z)=0$, and $I \in i^{*} \operatorname{Spec} \overline{R(\mathbf{Z}(S))}\left[t^{-1}\right]$, for some $g \in A$ with $\sigma(g)=f$. Notice that $\overline{R(\mathbf{Z}(S))}\left[t^{-1}\right]$ is a subring of $\overline{\mathbf{Z}(S)}((t))$, where $\overline{\mathbf{Z}(S)}$ is a completion of the filtered $\operatorname{ring} \mathbf{Z}(S)=\mathbf{Z}(A)_{g}$ with respect to negative degree subspaces, meaning that $\overline{\mathbf{Z}(S)}$ is the inverse limit $\mathbf{Z}(S) / \mathbf{Z}(S)_{n}$ as $n \rightarrow-\infty$, where $\mathbf{Z}(S)_{n}$ denotes the $n$-th degree filtration subspace of $\mathbf{Z}(S)$. More precisely, elements of $\overline{R(\mathbf{Z}(S))}\left[t^{-1}\right]$ are of the form $\sum_{i}^{\infty} s_{i} t^{i}$, where $s_{i} \in \overline{\mathbf{Z}(S)}$ such that the limit of $i-\operatorname{deg}\left(s_{i}\right)$ is $\infty$ as $i \rightarrow \infty$, and $s_{i}=0$ for sufficiently small $i \ll 0$. Thus, if $j: \mathbf{Z}(S) \rightarrow \overline{\mathbf{Z}(S)}$ denotes the embedding and if $I \in \operatorname{Im}\left(j^{*}\right), j^{*}$ : $\operatorname{Spec}(\overline{\mathbf{Z}(S)}) \rightarrow \operatorname{Spec}(\mathbf{Z}(S))$, then $I$ belongs to the Azumaya locus. Invertible elements of $\overline{\mathbf{Z}(S)}$ are precisely those elements whose principal symbol is a power of $f$ times an element of $\mathbf{k}^{*}$. It follows from the following trivial lemma that a prime ideal $I$ of height 1 is in the image of $j^{*}$ if and only if $I$ contains no elements whose principal symbol is a power of $f$ up to an element of $\mathbf{k}^{*}$.

Lemma 2.1. Let $j: R \rightarrow S$ be an embedding of integral domains, and let $I$ be a prime ideal of height 1 of $R$ which belongs to the smooth locus of Spec $R$. If $I \cap S^{*}=\emptyset$, then $I \in \operatorname{Im}\left(j^{*}\right): \operatorname{Spec} S \rightarrow \operatorname{Spec} R$.

Proof. Without loss of generality we may assume that $R$ is a local ring with $I$ being the maximal ideal. Therefore, $I=(g)$ for some $g \in R$. Since $g \notin S^{*}$, there is a prime ideal $J \in \operatorname{Spec} S$ such that $g \in J$. Therefore, $I=j^{-1}(J)$.

Now what we want follows from the following well-known fact.

Lemma 2.2. Let $B$ be a nonnegatively filtered finitely generated commutative algebra over $k$, and let $I \subset B$ be an ideal. Then $h t(I)=h t(\operatorname{gr} I)$. 
Proof. We have that the Gelfand-Kirillov dimension of $B / I$ equals that of gr $B / \operatorname{gr} I=\operatorname{gr}(B / I)$, which implies that $h t(I)=h t(\operatorname{gr} I)$, since $\operatorname{dim} B=\operatorname{dim} \operatorname{gr} B$.

Note that if Spec gr $A$ consists of finitely many symplectic leaves, and $\operatorname{gr} \mathbf{Z}(A)=$ $(\operatorname{gr} A)^{p}$, then the assumptions of Theorem 2.2 are satisfied.

We will also use the following.

Lemma 2.3. Let $M$ be a finitely generated positively filtered module over a positively filtered commutative algebra $H$, such that gr $H$ is a domain and $\operatorname{gr} M$ is finite over gr $H$. Then the rank of $M$ over $H$ is equal to the rank of gr $M$ over gr $H$.

Proof. First of all, it is easy to see that the rank of gr $M$ over gr $H$ is $\leq \operatorname{rank}_{H} M$. Indeed, if $x_{1}, \ldots, x_{n} \in H$ are elements such that their principal parts $\sigma\left(x_{1}\right), \cdots, \sigma\left(x_{n}\right)$ $\in \operatorname{gr} M$ are gr $H$-linearly independent, then $x_{1}, \ldots, x_{n}$ are $H$-linearly independent in $M$. Now let us consider $R(M)$, the Rees module of $M$ over the Rees algebra $R(H) . R(M)$ is a finitely generated $R(H)$-module. From the fact that $R(M) /(t-\lambda)=M, \lambda \neq 0, R(M) / t R(M)=$ gr $M$ we see that the generic dimension of fibers of $R(M)$ over $R(H)$ has dimension equal to $\operatorname{rank}_{H} M$. Therefore, by the semi-continuity, we conclude that $\operatorname{rank}_{\mathrm{gr} H}$ gr $M \geq \operatorname{rank}_{H} M$, so we are done.

Lemma 2.4. Suppose that an algebra $A$ satisfies all the assumptions of Theorem 2.2, except that $\operatorname{gr} \mathbf{Z}(A)=(\operatorname{gr} A)^{p}$. If $\operatorname{gr} A$ is normal, then $\operatorname{gr} \mathbf{Z}(A) \subset(\operatorname{gr} A)^{p}$.

Proof. Let $f \in \mathbf{Z}(A)$. Then its top symbol $\bar{f}$ belongs to the Poisson center of gr $A$. Let $U \subset X$ be the smooth locus of $X$. By the assumption, $U$ is a symplectic variety and $\bar{f} \mid U$ belongs to the Poisson center of $O(U)$. Therefore, $\left.d f\right|_{U}=0$; hence, there exists $g \in O(U)$, such that $\left.\bar{f}\right|_{U}=g^{p}$. Since $X-U$ has codimension $\geq 2$ in $X$ and $X$ is normal, $g$ extends to the whole $X$. Thus, $\bar{f} \in(\operatorname{gr} A)^{p}$.

Theorem 2.3. Let $A$ be an algebra over $\mathbf{k}$ equipped with an increasing algebra filtration $\mathbf{k}=A_{0} \subset A_{1} \subset \cdots \subset A_{n} \subset \cdots$, such that $\operatorname{gr} A$ is a finitely generated smooth commutative domain over $\mathbf{k}$. Suppose that there exist a central subalgebra of $A, \mathbf{Z}_{0} \subset \mathbf{Z}(A)$, such that $\operatorname{gr} A /(\operatorname{gr} A)\left(\operatorname{gr} Z_{0}\right)_{+}$is a domain whose smooth locus is a symplectic variety under the natural Poisson bracket and whose complement has codimension $\geq 2$. Assume moreover that $\operatorname{gr} A /(\operatorname{gr} A)\left(\operatorname{gr} \mathbf{Z}_{0}\right)_{+}$is normal. If $(\operatorname{gr} A)^{p} \subset \operatorname{gr} \mathbf{Z}(A)$, then the smooth and the Azumaya loci of $A$ coincide, and the PI-degree of $A$ is $p^{d}$, where $2 d=\operatorname{dim}(\operatorname{gr} A) /(\operatorname{gr} A)\left(\operatorname{gr} \mathbf{Z}_{0}\right)_{+}$.

Proof. Let $\chi_{0}: \mathbf{Z}_{\mathbf{0}} \rightarrow \mathbf{k}$ be a character. Then we can consider the quotient algebra $A_{\chi_{0}}=A \otimes \mathbf{z}_{0} \mathbf{k}$. Then, $A_{\chi_{0}}$ comes equipped with the natural filtration induced from $A$ and gr $A_{\chi_{0}}=\operatorname{gr} A /\left(\operatorname{gr} \mathbf{Z}_{0}\right)_{+}$. Also, Lemma 2.4 with the assumption $\operatorname{gr} \mathbf{Z}(A) \subset(\operatorname{gr} A)^{p}$ implies that $\left.\operatorname{gr} \mathbf{Z}\left(A_{\chi_{0}}\right)=\left(\operatorname{gr} A /\left(\operatorname{gr} \mathbf{Z}_{\mathbf{0}}\right)_{+}\right)\right)^{p}$. Thus, we may apply our proposition, which implies that the Azumaya locus of $\mathbf{Z}\left(A_{\chi_{0}}\right)$ has complement of codimension at least 2. Now we claim that a character $\chi: \mathbf{Z}(A) \rightarrow \mathbf{k}$ belongs to the Azumaya locus of $\mathbf{Z}(A)$ if and only if the corresponding character $\chi_{0}: \mathbf{Z}\left(A_{\chi_{0}}\right) \rightarrow \mathbf{k}$ belongs to the Azumaya locus of $A_{\chi_{0}}$, where $\chi_{0}$ is the restriction of $\chi$ on $\mathbf{Z}_{\mathbf{0}}$. It is enough to check that the PI-degree of $A$ is equal to the PI-degree of $A_{\chi_{0}}$ for any character $\chi_{0}: \mathbf{Z}_{\mathbf{0}} \rightarrow \mathbf{k}$. But this is clear because the PI-degree of $A_{\chi_{0}}$, which is equal to the square root of the dimension of the generic fiber of $A_{\chi_{0}}$ ([BG]), is greater than or equal to the PI-degree of $A$ (the square root of the dimension of the 
generic fiber of $A$ ) by the semi-continuity of the rank of fibers of $A$. On the other hand, the PI-degree of $A$ is the largest dimension of an irreducible module of $A$, which is greater than or equal to the largest possible dimension of an irreducible module of $A_{\chi_{0}}$, which is precisely the PI-degree of $A_{\chi}$.

So, let $U \subset \operatorname{Spec} \mathbf{Z}(A)$ be the Azumaya locus of $A$. We have a map $f$ : $\operatorname{Spec} \mathbf{Z}(A) \rightarrow$ Spec $\mathbf{Z}_{\mathbf{0}}$ corresponding to the inclusion $\mathbf{Z}_{\mathbf{0}} \subset \mathbf{Z}(A)$. Let us denote by $Y$ the complement of the Azumaya locus $Y=\operatorname{Spec} \mathbf{Z}(A)-U$. Then for any closed point $\chi \in \operatorname{Spec} \mathbf{Z}_{\mathbf{0}}$, the intersection $f^{-1}(\chi) \cap Y$ has codimension at least 2 in $f^{-1}(\chi)$. So, the codimension of $Y$ in $\operatorname{Spec} \mathbf{Z}(A)$ is at least 2. Now, since $A$ is Auslander-regular and Cohen-Macaulay, the above-mentioned result of BrownGoodearl $[\mathrm{BG}$ ] implies the coincidence of the Azumaya and smooth loci.

By Lemma 2.3, the PI degree of $A$ is equal to the rank of $\operatorname{gr} A /(\operatorname{gr} A)\left(\operatorname{gr} \mathbf{Z}_{\mathbf{0}}\right)_{+}$ over $\left(\operatorname{gr} A /(\operatorname{gr} A)\left(\operatorname{gr} \mathbf{Z}_{\mathbf{0}}\right)_{+}\right)^{p}$, which is $p^{d}$, where $2 d$ is the Krull dimension of gr $A$ / (gr $A)\left(\operatorname{gr} \mathbf{Z}_{\mathbf{0}}\right)_{+}$.

\section{Applications to Symplectic Reflection Algebras AND ENVELOPING ALGEBRAS}

Let us recall the definition of a symplectic reflection algebra. Let $V$ be a symplectic k-vector space with the symplectic form $\omega: V \times V \rightarrow \mathbf{k}$. An element $g \in S p(V)$ is called a symplectic reflection if $\operatorname{rank}(I d-g)=2$. To a symplectic reflection $s \in S p(V)$ one may associate a skew-symmetric form $\omega_{s}: V \times V \rightarrow \mathbf{k}$ which coincides with $\omega$ on $\operatorname{Im}(I d-s)$ and is 0 on $\operatorname{Ker}(I d-s)$. Let $G \subset S p(V)$ be a finite group generated by symplectic reflections. To a $G$-invariant function $c: S \rightarrow \mathbf{k}$ and $t \in \mathbf{k}$, where $S \subset G$ is the subset of symplectic reflections of $G$, Etingof and Ginzburg [EG] associated an algebra (called a symplectic reflection algebra) $H_{t, c}$ which is defined as a quotient of $\mathbf{k}[G] \ltimes T(V)$ by the relations

$$
[x, y]=t \omega(x, y)+\sum_{s \in S} \omega_{s}(x, y) c(s) s .
$$

There is a filtration on $H_{t, s} \operatorname{deg} v=1, v \in V, \operatorname{deg} g=0, g \in G$. The crucial property is that gr $H_{t, c}=\mathbf{k}[G] \ltimes \operatorname{Sym} V$ [EG].

The following theorem answers positively two questions raised by Brown and Changtong ([BC], Questions 6.1,6.4), and proved by them in the case of a rational Cherednik algebra.

Theorem 3.1. Let $H_{t, c}$ be a symplectic reflection algebra associated to $G \subset S p(V)$, $\operatorname{dim} V=2 n, t \neq 0$ and $p$ does not divide the order of $G$. Then the smooth and the Azumaya loci of the center of $H_{t, c}$ coincide, and the PI-degree of $H_{t, c}$ (the maximal dimension of an irreducible module) is equal to $p^{n}|G|$.

Proof. Let us consider $U_{t, c}=e H_{t, c} e$, the spherical subalgebra of the symplectic reflection algebra $H_{t, c}$, where $e=\frac{1}{|G|} \sum_{g \in G} g$ is the symmetrizing idempotent of $G$. By a theorem of Etingof BFG], $\operatorname{gr} \mathbf{Z}\left(U_{t, c}\right)=\left((\operatorname{Sym} V)^{G}\right)^{p}=\left(\operatorname{gr} U_{t, c}\right)^{p}$. Theorem 2.2 can be applied to $U_{t, c}$, since gr $U_{t, c}=S(V)^{G} \subset S y m V$ has finitely many symplectic leaves by a result of Brown-Gordon ( $\mathrm{BGO}$, Proposition 7.4). So, we get that for all prime ideals $I$ of the smooth locus of $\mathbf{Z}\left(U_{t, c}\right)$ of height 1 , the algebra $U_{t, c}$ is Azumaya. But by a result of Brown-Changtong ( $\mathrm{BC}$, Corollary 3.6), $\left(H_{t, c}\right)_{I}$ is Morita equivalent to $\left(U_{t, c}\right)_{I}$, so the complement of the Azumaya locus in the smooth locus of $H_{t, c}$ has codimension $\geq 2$. Since $H_{t, c}$ is Auslander-regular and 
Cohen-Macaulay $([\mathrm{BC}])$, by Brown-Goodearl $[\mathrm{BG}]$, the smooth and the Azumaya loci coincide for $\mathbf{Z}\left(H_{t, c}\right)$. Lemma 2.3 applied to $M=H_{t, c}, H=\mathbf{Z}\left(H_{t, c}\right)$ implies that the PI-degree is independent of $c$, so we may take $c=0$, in which case the desired statement is clear.

Applying the considerations from the previous section, we may take $A$ to be the enveloping algebra of a semi-simple Lie algebra $\mathfrak{g}$ and take $\mathbf{Z}_{\mathbf{0}} \subset \mathbf{Z}(A)$ to be the subalgebra obtained by the symmetrization map applied to the generators of $(\operatorname{Sym} g)^{G}$. In other words, $\mathbf{Z}_{\mathbf{0}}$ is the reduction modulo $p$ of the usual characteristic 0 central elements of $\mathfrak{U} \mathfrak{g}$. Then $\operatorname{gr} A /\left(\operatorname{gr} \mathbf{Z}_{\mathbf{0}}\right)_{+}=\operatorname{Sym} \mathfrak{g} /(\mathrm{Sym} \mathfrak{g})_{+}^{G}$ is the ring of coinvariants, which is the ring of functions on the nilpotent cone of $\mathfrak{g}^{*} \mathrm{Ko}$, which as a Poisson variety consists of finitely many symplectic leaves. Thus, the assumptions of Theorem 2.3 are satisfied. As a result we obtain that the Azumaya locus of $\mathfrak{U} \mathfrak{g}$ coincides with the smooth locus of its center, a theorem of Brown-Goodearl [BC]. Note that we did not use any modular representation theory of $\mathfrak{g}$.

A standard example of an almost commutative algebra for which the Azumaya locus does not coincide with the smooth locus is the enveloping algebra of the Heisenberg Lie algebra: A Lie algebra $\mathfrak{g}$ with a basis $z, x_{1}, \ldots, x_{n}, y_{1}, \ldots, y_{n}$ and relations $\left[x_{i}, y_{j}\right]=\delta_{i j} z,\left[z, x_{i}\right]=\left[z, y_{j}\right]=0$, where $\delta_{i j}$ is the Kronecker symbol. Then, the center of $\mathfrak{U} \mathfrak{g}$ is the polynomial algebra $\mathbf{k}\left[z, x_{1}^{p}, \ldots, x_{n}^{p}, y_{1}^{p}, \ldots, y_{n}^{p}\right]$, but the Azumaya locus is the set of characters which do not vanish on $z$.

The above theorem can also applied to infinitesimal Hecke algebras of $\mathfrak{s l}_{2}[\mathrm{~T}$. We expect many applications of the above result for other infinitesimal Hecke algebras.

\section{ACKNOWLEDGEMENTS}

The author would like to thank M. Boyarchenko and especially V. Ginzburg for useful discussions. The author is also grateful to the anonymous referee for helpful comments.

\section{REFERENCES}

[BC] K. Brown and K. Changtong, Symplectic reflection algebras in positive characteristic, Proc. Edinb. Math. Soc. (2) 53 (2010), no. 1, 61-81. MR.2579679

[BG] K. Brown and K. Goodearl, Homological aspects of Noetherian PI Hopf algebras and irreducible modules of maximal dimension, Journal of Algebra 198 (1997), 240-265. MR 1482982 (99c:16036)

[BGo] K. Brown and I. Gordon, Poisson orders, symplectic reflection algebras and representation theory, J. Reine Angew. Math. 559 (2003), 193-216. MR1989650 (2004i:16025)

[BFG] R. Bezrukavnikov, M. Finkelberg, and V. Ginzburg, Cherednik algebras and Hilbert schemes in characteristic p, Represent. Theory 10 (2006), 254-298. MR2219114 (2007j:14004)

[BK] R. Bezrukavnikov and D. Kaledin, Fedosov quantization in positive characteristic, J. Amer. Math. Soc. 21 (2008), no. 2, 409-438. MR2373355 (2008i:14079)

[EG] P. Etingof and V. Ginzburg, Symplectic reflection algebras and Calogero-Moser spaces and deformed Harish-Chandra homomorphism, Inventiones Math. 147 (2002), no. 2, 243-348. MR.1881922 (2003b:16021)

[Ko] B. Kostant, Lie group representations on polynomial rings, Amer. J. Math. 85 (1963), 327-404. MR0158024 (28:1252)

[T] A. Tikaradze, Infinitesimal Hecke algebras of $\mathfrak{s l}_{2}$ in positive characteristic, J. Algebra 321 (2009), no. 1, 117-127. MR2469352 (2009i:17030)

Department of Mathematics, The University of Toledo, Toledo, Ohio 43606

E-mail address: atikara@utnet.utoledo.edu 